\title{
The influence of oxygen and nitrogen doping on GeSbTe phase-change optical recording media properties
}

\author{
D. Dimitrov*,1, H.-P.D. Shieh \\ Institute of Electro-Optical Engineering, National Chiao Tung University, 1001 Ta Hsueh Road, Hsinchu 3000, Taiwan, ROC
}

Received 12 May 2003

\begin{abstract}
Nitrogen and oxygen doped and co-doped GeSbTe (GST) films for phase-change optical recording are investigated. It is found that the crystallization temperature increased as well as the crystalline microstructure refined by doping. The carrier-to-noise ratio (CNR) and erasability of phase-change optical disks are improved being up to 52 and $35 \mathrm{~dB}$, respectively, by using an appropriate nitrogen doping or co-doping concentration in the recording layer. Optical disks with co-doped recording layer are found to be superior in the recording characteristics then the single doped recording layer disks.
\end{abstract}

(c) 2003 Elsevier B.V. All rights reserved.

Keywords: Optical data storage; Phase-change media; GeSbTe

\section{Introduction}

The phase-change optical recording technology utilizes the reversible structural change between amorphous and crystalline state of certain materials mainly based on chalcogenide alloys and compounds [1]. GeSbTe system materials $[2,3]$ possessing both the amorphous state stability and high crystallization speed are found to be very suitable for application in phase-change optical recording.

The most important goals in the research of phase-change optical recording are a continuous increase of the recording density, data rate and overwrite cyclability [4]. It is however highly desirable these improvements to be achieved using disks with a simpler stack structure. The recording layer material composition optimization by means of reactive sputtering is a common method for achieving better disk performance. It was reported that nitrogen [5] and oxygen [6] addition to GeSbTe (GST) recording material increases overwritability and effectively prevents the initial jitter increases in phase change optical recording disks. However, since these two gases possess very different reactivity, it is rather difficult to control the doping concentration in the film

\footnotetext{
* Corresponding author. Tel.: +886-3-5913574; fax: +886-3-5820442. E-mail address: Dimitre2000@excite.com (D. Dimitrov).

${ }^{1}$ On leave from Central Laboratory of Photoprocesses, Bulgarian Academy of Sciences, 1113 Sofia, Bulgaria.
}

and/or the sputtering rate. In our previous study [7], we use co-doping in order to combine the influence of both oxygen and nitrogen on films physical properties and recording media performance. The goal of present research article is to compare the effect of single oxygen or nitrogen doping and double doping on GeSbTe recording medium properties.

\section{Experimental details}

GST films containing oxygen or nitrogen and both, denoted hereinafter as GSTO, GSTN, and GSTON films respectively, were prepared by reactive RF magnetron sputtering of $\mathrm{Ge}_{21} \mathrm{Sb}_{26} \mathrm{Te}_{53}$ target in the presence of oxygen and/or nitrogen as reactive gases in the sputtering chamber. The crystallization temperatures $\left(T_{\mathrm{x}}\right)$ were obtained from the reflection changes monitoring during constant rate $(50 \mathrm{~K} / \mathrm{min})$ heating. The films were annealed at $473 \mathrm{~K}$ for $20 \mathrm{~min}$ for X-ray diffraction (XRD) measurements. The measurements were performed on a diffractometer operated at $40 \mathrm{kV}$ and $40 \mathrm{~mA} . \mathrm{Cu} \mathrm{K}_{\alpha}$ radiation with $\lambda=1.54056 \AA$ has been used. Data were collected in Bragg-Brentano mode and grazing incidence angle of $1^{\circ}$ within the range of $2 \Theta$ from $20^{\circ}$ to $60^{\circ}$. Simple quadrilayer disk structures $\mathrm{ZnS}-\mathrm{SiO}_{2} /$ doped-GeSbTe/ZnS-SiO $/ 2 / \mathrm{Al}$ alloy were consecutively sputter-deposited onto pre-grooved polycarbonate 
disk substrates for dynamic testing experiments. The dynamic testing evaluation of the recording/erasing characteristics was performed using a laser tester with a wavelength of $660 \mathrm{~nm}$ and a $0.6 \mathrm{NA}$ lens at a disk rotation speed of $5.8 \mathrm{~m} / \mathrm{s}$.

\section{Results and discussion}

\subsection{Crystallization temperatures}

Fig. 1 shows the reflectivity changes during constant rate heating of a number of GeSbTe co-doped films. Oxygen and nitrogen flow contents during sputtering expressed in cubic centimeters per minute ( $\mathrm{sccm}$ ) at Ar flow of $100 \mathrm{sccm}$ and $T_{\mathrm{x}}$ of as-deposited films are also shown in Fig. 1. $T_{\mathrm{x}}$, measured as the temperature at the middle of the sharp reflectivity drop during heating, increases gradually from 410 to $448 \mathrm{~K}$ with increase of the co-doping gases concentration. The crystallization temperature dependence on the doping content in thin films is shown in Fig. 2. The crystallization temperature increases almost proportionally to the doping concentration. Oxygen is more effective for increasing of the $T_{\mathrm{x}}$ probably due to the higher reactivity and melting point and crystallization temperature of oxides formed in the films. It is widely supposed [8] that in general the long-term stability of the amorphous phase against crystallization increases with increased melting temperature. The crystallization temperature on the other side is found empirically to fall in the range of $1 / 3$ and $2 / 3$ the melting temperature [9]. It was furthermore established [10] that materials, which crystallize at more than $423 \mathrm{~K}(10 \mathrm{~K} / \mathrm{min}$ heating rate $)$ are preferable to ensure archival stability of the amorphous data. Finally the

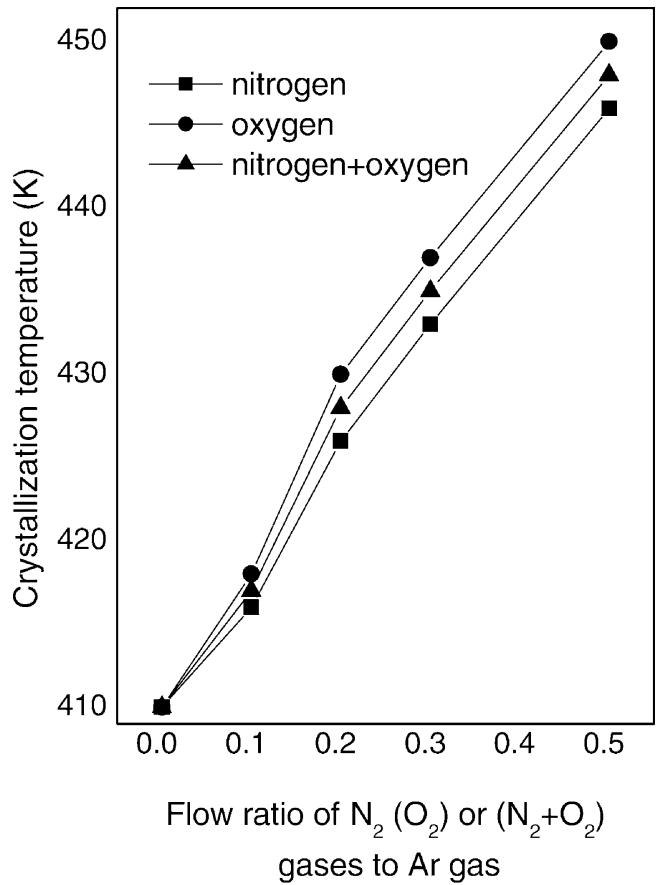

Fig. 2. Crystallization temperatures dependence on the flow ratio of $\mathrm{N}_{2}$ $\left(\mathrm{O}_{2}\right)$ or $\left(\mathrm{N}_{2}+\mathrm{O}_{2}\right)$ gases to Ar gas.

glass transition temperature $T_{\mathrm{g}}$, which is a measure for the stability of the amorphous state, is typically $50-70 \%$ of $T_{\mathrm{x}}$.

\subsection{Film structure}

XRD measurements are shown in Fig. 3. Few lines are visible for either of the thin films, indicating high symmetry

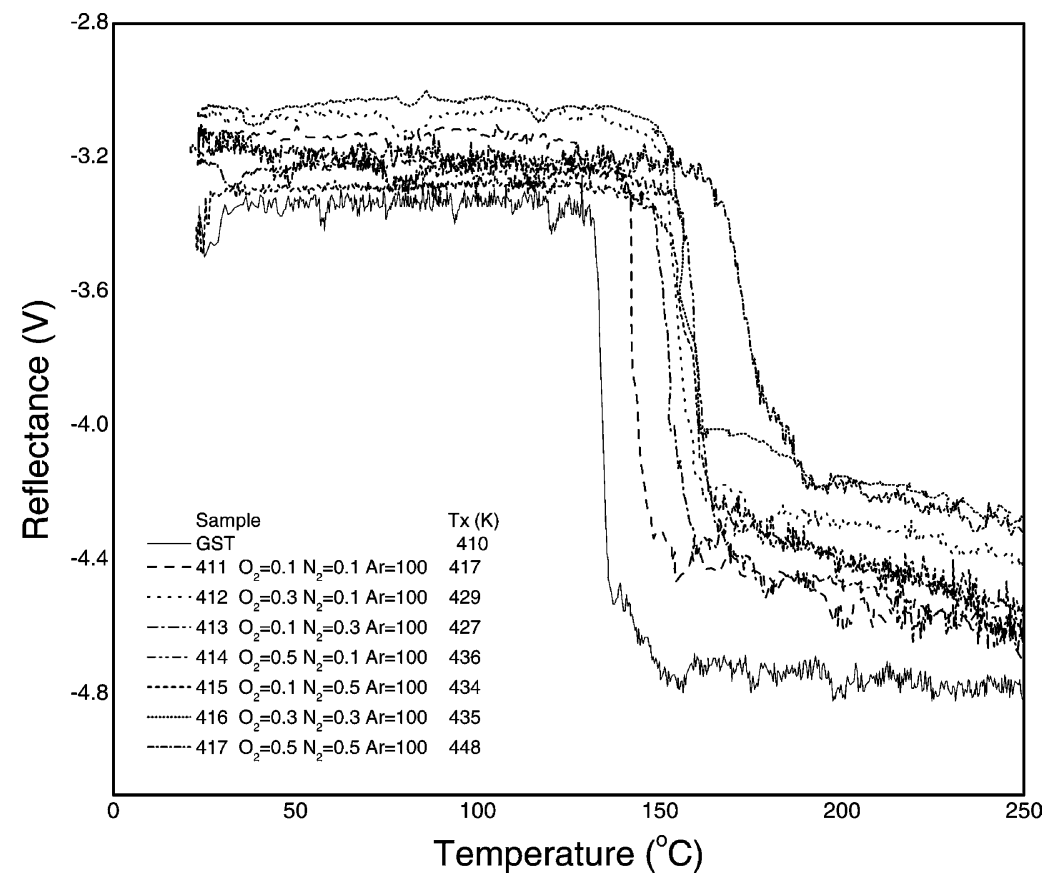

Fig. 1. Reflectance changes during constant rate heating for determination of the crystallization temperatures of doped GeSbTe films. 


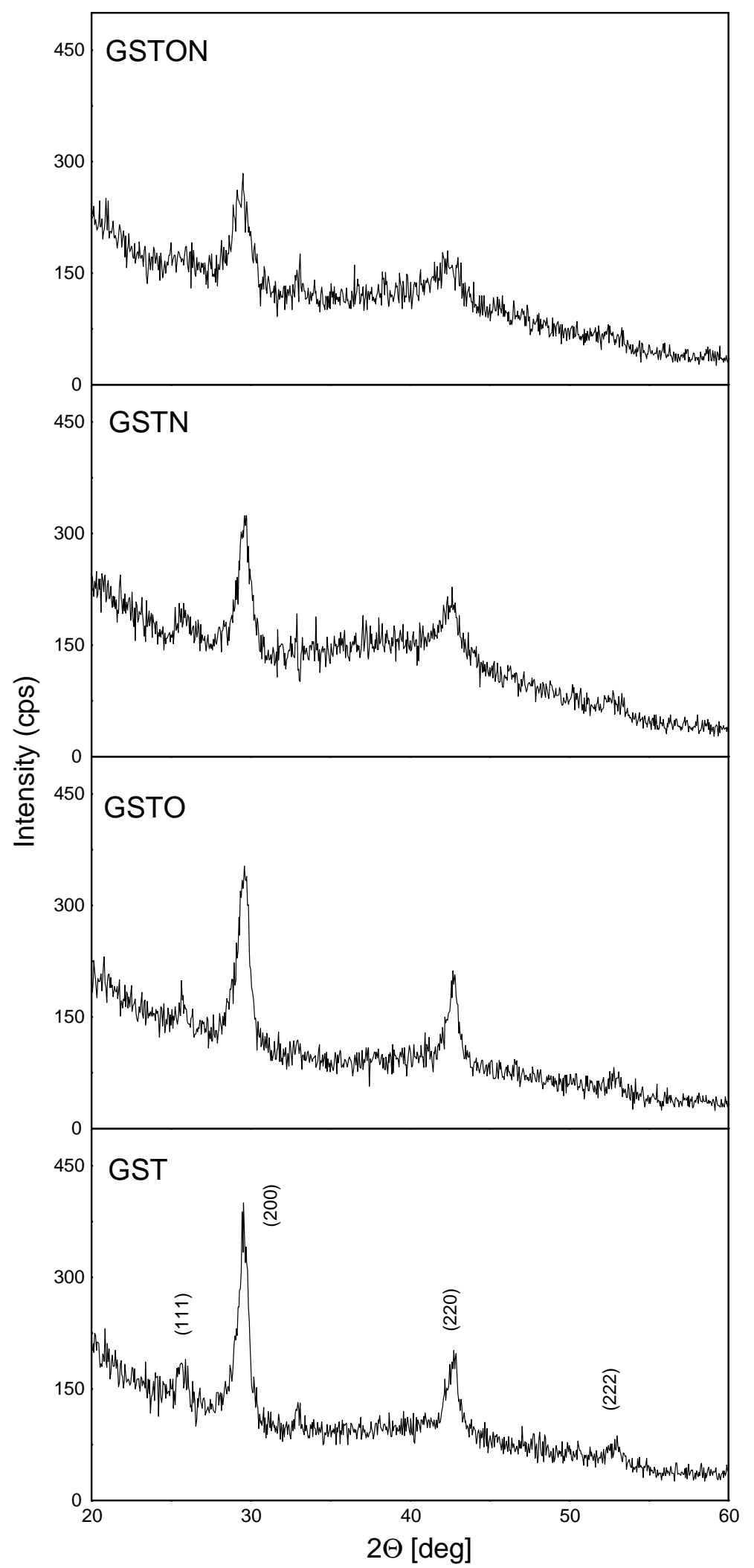

Fig. 3. X-ray diffraction (XRD) patterns of crystallized GST, GSTO, GSTN, and GSTON thin films. 
in the lattice. The crystal structures of GST, GSTO, GSTN, and GSTON after annealing are similar to each other and are best described using a face-centered cubic (fcc) lattice symmetry. These simple structures with a high degree of symmetry are believed to be prerequisite for a rapid crystallization [11]. Moreover, in a system having a high degree of symmetry most positions in the crystal lattice are equivalent. Since constituent atoms can occupy almost any lattice position the atomic motion needed for a phase change is reduced thus the crystallization speed increases. The line widths increase with doping suggesting a grain refinement in doped films. We can assume that doped films probably possess a higher number of nuclei during crystallization than GST.

\subsection{Measurements of the optical disks recording characteristics}

Recording characteristics of several disks with different recording layer compositions prepared using the sputtering conditions shown in Table 1 were measured. Accordingly, the disks were labeled as GST, GSTO, GSTN, and GSTON (411, 412, 413, and 416). The carrier-to-noise ratio (CNR) dependence for $1.5 \mathrm{~T}$ marks on the peak writing power for disks with different oxygen and nitrogen concentration (respectively, different $\mathrm{O}_{2}+\mathrm{N}_{2} / \mathrm{Ar}$ ratio during reactive sputtering of a GeSbTe target) in the recording layer is shown on Fig. 4. CNR of disks with oxygen and nitrogen doping is comparable with those of GST disk. Moreover using a very small co-doping (disk sample 411, see Table 1), it is possible to increase CNR up to $52 \mathrm{~dB}$. Technologically important is also the increased power range for obtaining a maximal CNR in oxygen and nitrogen co-doped disks. Furthermore the threshold power for obtaining the adequate CNR level, for instance $45 \mathrm{~dB}$, is clearly reduced in most of the doped disks. Fig. 5 shows the dependence of erasability on the bias power. Measurements were carried out after repeating alternate overwrites of 3 and $11 \mathrm{~T}$ marks. The erasability of co-doped disks is appreciated higher than in those GST disks. Up to $5 \mathrm{~dB}$ increases of the erasability was obtained in co-doped disk 411. The threshold bias power for obtaining a maximal erasability also decreases with co-doping.

Table 1

Sputtering conditions for preparation of GeSbTe film doped with oxygen, nitrogen and co-doped with both oxygen and nitrogen

\begin{tabular}{llll}
\hline Sample ID & $\mathrm{O}_{2} / \mathrm{Ar}(\mathrm{sccm})$ & $\mathrm{N}_{2} / \mathrm{Ar}(\mathrm{sccm})$ & $\mathrm{O}_{2}+\mathrm{N}_{2} / \mathrm{Ar}(\mathrm{sccm})$ \\
\hline GST & - & - & - \\
GSTO & $0.2 / 100$ & - & - \\
GSTN & - & $0.2 / 100$ & - \\
GSTON(411) & - & - & $0.1+0.1 / 100$ \\
GSTON(412) & - & - & $0.3+0.1 / 100$ \\
GSTON(413) & - & - & $0.1+0.3 / 100$ \\
GSTON(414) & - & - & $0.5+0.1 / 100$ \\
GSTON(415) & - & - & $0.1+0.5 / 100$ \\
GSTON(416) & - & - & $0.3+0.3 / 100$ \\
GSTON(417) & - & - & $0.5+0.5 / 100$ \\
\hline
\end{tabular}

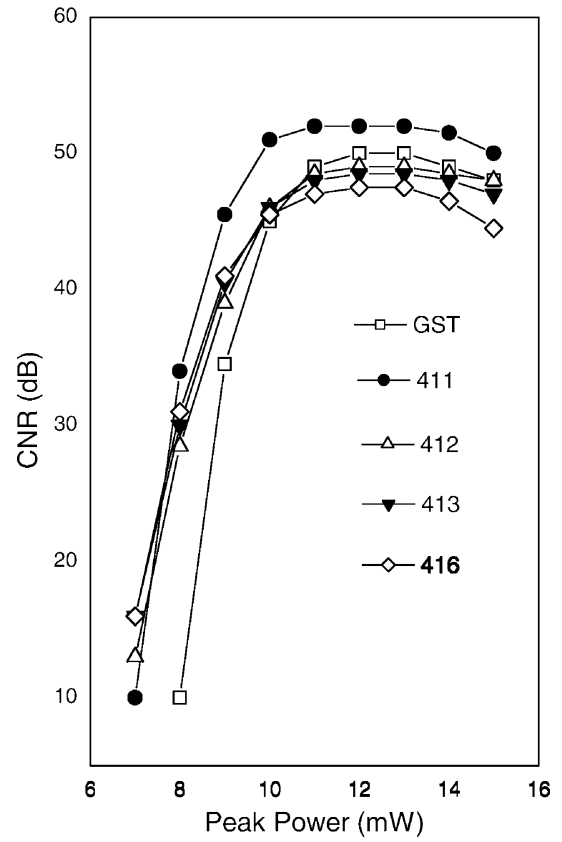

Fig. 4. CNR dependence on peak power for disks with nitrogen and oxygen co-doped recording layer.

Next the CNR and erasability characteristics of disks using single oxygen or nitrogen doping were compared with those of double-doped disk. The measurements conditions were the same as stated above. It is clearly seen from Figs. 6 and 7 that nitrogen and oxygen doping and co-doping lead to improvement of the recording/erasing parameters of the disks. The CNR and erasability behavior on the laser power for the nitrogen doped and co-doped disks

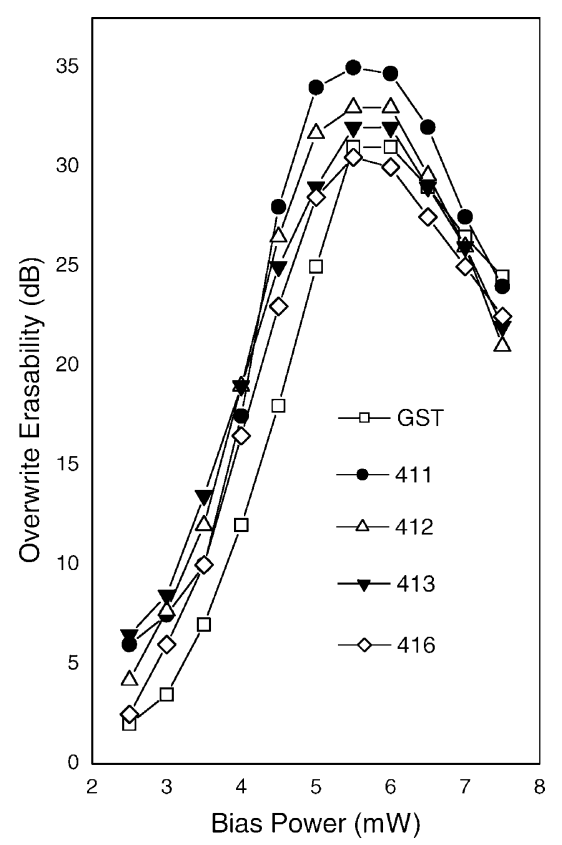

Fig. 5. Erasability dependence on the bias power for disks with nitrogen and oxygen co-doped recording layer. 


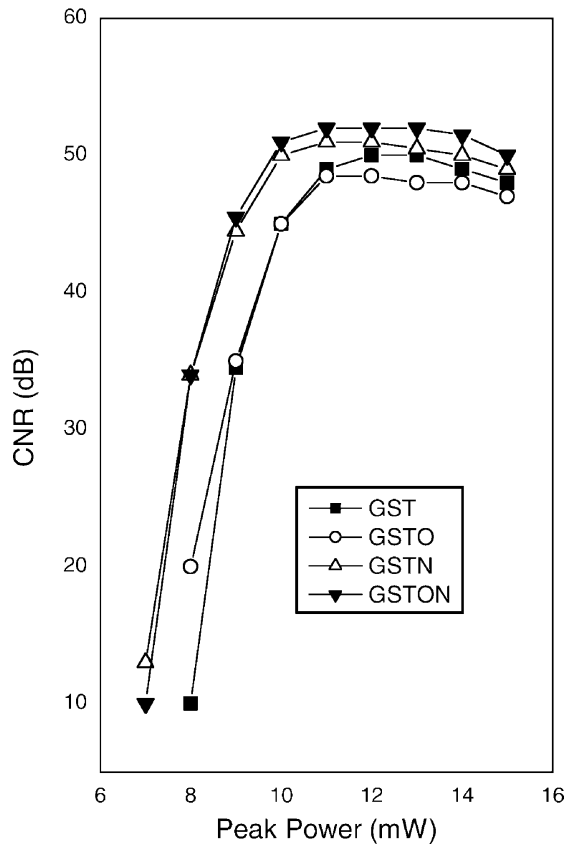

Fig. 6. CNR dependence on the peak power for disks with different doping in the recording layer.

are nearly the same. However, both CNR and erasability as well as the necessary peak power and bias power for obtaining the suitable levels of the CNR and erasability of GSTON disk (411) are slightly superior then those in the single doped disks. Hence by using a small co-doping concentration the recording characteristics of the disks can be improved even further in comparison with the single doped recording layer disks.

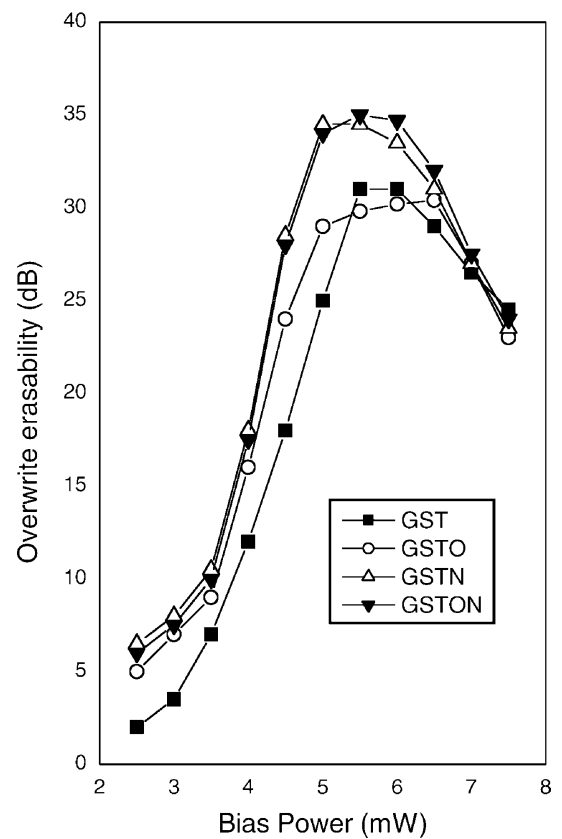

Fig. 7. Erasability dependence on the bias power for disks with different doping in the recording layer.
The reduction in threshold peak writing power and bias power could be associated with increasing of the recording sensitivity [12] by oxygen and nitrogen co-doping. It is supposed [13] that nitrides and oxides are formed in the co-doped material and they reduced the thermal conductivity of the recording layer leading to improved recording and erasing sensitivity. Furthermore small doping fraction is effective in increasing the crystallization speed most probably due to the enhanced nucleation rate. Indeed transmission electron microscopy (TEM) and X-ray diffraction (XRD) measurements [7,14] show that the number of crystal grains increase while the crystal size decreases as the nitrogen or co-doping content increases. The opposite tendency however is reported [15] for GeSbTe films doped with oxygen. It is supposed that nitrides and/or oxides condensed at the grain boundaries of the GeSbTe microcrystals thus forming a thin film. During the melting for amorphization these nitrides/oxides, usually having high melting points, remain in a solid state and suppress the micro material flow phenomena in GeSbTe. Nitrides and oxides have lower thermal conductivity than GeSbTe and the overall thermal conductivity of the recording layer decreases with nitrogen/oxygen concentration. This will lead to suppression of the heat diffusion produced by laser irradiation of the recording layer and increase in the sensitivity of recording and erasing. The function of nitrogen and oxygen atoms in the recording layer and the mechanism of recording/erasing characteristics improvement as proposed in [12] and [14] could also explain the effect of co-doping observed in our experiments.

\section{Conclusions}

Oxygen and nitrogen doped and co-doped GST films possess higher $T_{\mathrm{x}}$ than undoped GST and fine crystalline microstructure, which are beneficial for phase-change optical recording applications. Recording characteristics of optimized disk structures are being measured using dynamic tester and it is found that recording sensitivity and erasability are improved by co-doping of oxygen and nitrogen into GeSbTe recording layer, while the CNR being almost the same for nitrogen doped and co-doped disks. Hence the co-doped recording layer disks possess superior characteristics than the disks with single oxygen or nitrogen doping in the recording layer. The possibility for further improvements exists by additional optimization of the sputter-deposition process, recording layer composition and disk multilayer structure.

\section{Acknowledgements}

Thanks are due to Mrs. M.-R. Tseng and Mr. W.-C. Hsu both from MRL, ITRI, Taiwan for the crystallization temperature measurements. This work was supported by National 
Science Council of Taiwan under the contract "Photonics Science and Technology for Tera Era” no. 89-E-FA06-1-4.

\section{References}

[1] G.F. Zhou, Mater. Sci. Eng. A 304-306 (2001) 73-80.

[2] N. Yamada, E. Ohno, N. Akahira, K. Nishiuchi, K. Nagata, M. Takao, Jpn. J. Appl. Phys. 26 (1987) 61-66.

[3] N. Yamada, E. Ohno, K. Nishiuchi, N. Akahira, M. Takao, J. Appl. Phys. 69 (1991) 2849-2856.

[4] N. Yamada, Proc. SPIE 3109 (1997) 28-37.

[5] R. Kojima, T. Kouzaki, T. Matsunaga, N. Yamada, Proc. SPIE 3401 (1998) 14-23.
[6] G.-F. Zhou, B.A.J. Jacobs, Jpn. J. Appl. Phys. 38 (1999) 1625-1628.

[7] D.Z. Dimitrov, Y.-H. Lu, M.-R. Tseng, W.-C. Hsu, H.-P.D. Shieh, Jpn. J. Appl. Phys. 41 (2002) 1656-1659.

[8] K.A. Rubin, Mat. Res. Soc. Symp. Proc. 230 (1992) 239-250.

[9] K.A. Rubin, M. Chen, Thin Solid Films 181 (1989) 129-139.

[10] K.A. Rubin, J. Magn. Soc. Jpn. 15 (1991) 127-132.

[11] N. Yamada, T. Matsunaga, J. Appl. Phys. 88 (2000) 7020-7028.

[12] R. Kojima, S. Okabayashi, T. Kashihara, K. Horai, T. Matsunaga, E. Ohno, N. Yamada, T. Ohta, Jpn. J. Appl. Phys. 37 (1998) 2098 2103.

[13] H.J. Borg, J.P.W.B. Duchateau, Proc. SPIE 3109 (1997) 20-25.

[14] T.H. Jeong, M.R. Kim, H. Seo, J.W. Park, C. Yeon, Jpn. J. Appl. Phys. 39 (2000) 2775-2779.

[15] A. Ebina, M. Hirasaka, K. Nakatani, J. Vac. Sci. Technol. A 17 (1999) 3463-3466. 